\title{
Does a Hospital Palliative Care Team Have the Potential to Reduce the Cost of a Terminal Hospitalization? A Retrospective Case-control Study in a Czech Tertiary University Hospital
}

\section{Zuzana Kremenova ( $\nabla$ zuzana.kremenova@fnkv.cz)}

Faculty Hospital Kralovske Vinohrady and Third Faculty of Medicine, Charles University, Prague, Czech Republic

\section{Jan Svancara}

Institute of Health Information and Statistics of the Czech Republic, Prague, Czech Republic

\section{Petra Kralova}

Faculty Hospital Kralovske Vinohrady and Third Faculty of Medicine, Charles University, Prague, Czech

Republic

\section{Martin Moravec}

Institute for Medical Humanities, First Faculty of Medicine, Charles University, Prague, Czech Republic

\section{Katerina Hanouskova}

Faculty Hospital Kralovske Vinohrady and Third Faculty of Medicine, Charles University, Prague, Czech Republic

\section{Mayara Knizek-Bonatto}

Faculty Hospital Kralovske Vinohrady and Third Faculty of Medicine, Charles University, Prague, Czech Republic

\section{Research Article}

Keywords: palliative care, hospital, cost savings, end of life, medical records

Posted Date: June 23rd, 2021

DOl: https://doi.org/10.21203/rs.3.rs-625443/v1

License: (c) (i) This work is licensed under a Creative Commons Attribution 4.0 International License. Read Full License 


\section{Abstract}

Background: More than $50 \%$ of patients worldwide die in hospitals. It is well known that end-of-life hospital care is costly.

Our aims were to test whether the support of the palliative team can reduce end-of-life costs and to study the mechanisms of cost reduction.

Methods: This was a one-centre descriptive retrospective case-control study. Big data from registries of routine visits were used for case-control matching. We included the expenses billed to the insurance company and added separately charged drugs and materials. We compared the groups over the duration of the terminal hospitalization, ICU days (ICU=intensive care unit), IV antibiotic use (IV=intravenous), $\mathrm{MRI} / \mathrm{CT}$ scans (MRI/CR=magnetic resonance imaging/computed tomography), oncologic treatment in the last month of life, and documentation of the dying phase.

We searched for all in-hospital cases who died in the university hospital in Prague with the support of the hospital palliative team from January 2019 to April 2020 and matched them with similar controls. The controls were matched according to age, sex, Charlson comorbidity index and diagnosis recorded on the death certificate.

Results: We identified 213 dyads. The average daily costs were three times lower in the palliative group (4,392.4 CZK per day=171.3 EUR) than in the non-palliative group $(13,992.8 \mathrm{CZK}$ per day=545.8 EUR), and the difference was caused by the shorter time spent in the ICU (16\% vs $33 \%$ of hospital days). This was probably due to better documentation of the dying phase in the medical records.

Conclusions: To date, there are sparse hospital data available on the economic aspects of end-of-life care. We showed that the integration of the palliative care team in the dying phase can be cost saving. The evidence that hospital palliative care can save a substantial amount of money can be used to support the integration of palliative care in hospitals in middle- and low-income countries. A multicentre study with the same design is planned in the future to increase the strength of the results.

\section{Background}

Palliative care is developing rapidly in Czech hospitals. The first hospital palliative care consult teams were established in 2016 and play a leading role in implementing palliative care in hospital settings. The palliative care team of the Faculty Hospital Kralovske Vinohrady in Prague is one such team. The opportunities available to maintain and prolong life in modern medicine give rise to medical and ethical dilemmas involving patients reaching the end of life. Difficult questions arise regarding whether specific treatments and diagnostic procedures are appropriate and beneficial to these patients. Intensified therapeutic and diagnostic procedures are often performed during terminal hospitalization despite their burden on the patients, the limited clinical value and the substantial healthcare costs. ${ }^{1}$ Sometimes further treatment is withheld, and an existing treatment is discontinued at the end of life to prevent futile 
healthcare. ${ }^{2}$ However, healthcare provided to the dying person can also be very intense, and some studies demonstrate that costs are the highest in the dying phase. ${ }^{3}$ Integration of palliative care in hospitals is associated with not only clinical benefits but also economic impact. ${ }^{4}$ Total direct hospital costs can be reduced in cancer and noncancer patients when the patient is supported by a palliative care team. 5,6 Maintaining patients' autonomy at the end of life is not only a matter of making choices and medical decisions but also an active preparation for their death and allows them to state their preferences for the end of their life. ${ }^{7}$ Documentation of the patient's preferences and stating the code status early are associated with a less aggressive end of life. ${ }^{8}$ Advanced care documentation can also encourage end-oflife discussions and preparations. ${ }^{9}$ Family members and those close to the patient should be involved in end-of-life planning and supported by the staff.

\section{Methods}

Aims

1. To determine whether integration of the hospital palliative care team at the end of life can prevent the use of burdensome diagnostic and therapeutic procedures.

2. To prove that if the palliative care team is involved, it gives clearer context to the end of life, as the dying phase is documented.

3. To prove that integration of hospital palliative care teams at the end of life can prevent transitions to intensive care and lower healthcare costs.

\section{Design}

We used a case-control observational retrospective study design. The differences in the end-of-life between the two groups of patients with (palliative care group) and without (nonpalliative care group) the support of the hospital palliative care team were compared. We retrospectively analysed and documented data from paper and electronic medical records of the terminal hospitalization.

\section{Setting}

The Faculty Hospital Kralovske Vinohrady is a tertiary university hospital in Prague, with 1,200 beds serving a catchment area of approximately 300,000 inhabitants. Patients in the catchment area are the oldest of all Prague inhabitants.

The hospital palliative care team was established in 2016, and with 748 new patients per year (in 2020), it is the most efficient and one of the most advanced teams in the Czech Republic. This has led to the implementation and development of specialist hospital palliative care in the country. Nearly one-third of the patients indicated to the team die during their terminal hospitalization. There were approximately 1,100 deaths in the hospital, with $15-19 \%$ of dying patients supported by the palliative care team.

Participants 
All in-patients who died between January 2019 and April 2020 were eligible for the study. Cases were supported by the hospital palliative care team during the dying phase. They were matched with similar controls from all deceased patients not supported by the team. Routine big data from the National Death Registry and the National Registry of Hospital Activity were used for matching. Controls had similar diagnoses on the death certificate, sex, age group, and Charlson comorbidity index. Power analysis was performed to calculate the sample size large enough to demonstrate the presence of an economically relevant financial difference between the two groups.

Data collection

Data were collected from paper and electronic medical records. Three researchers, all physicians (2 internists and 1 oncologist), analysed the records of the deceased patients. They inserted the variables into a prepared template. When the semiqualitative data were analysed, content analysis of the written data was carried out according to an approved mechanism about which a consensus was reached by all researchers and the project manager. Economic analysis was performed by counting all hospital costs of health care procedures billed to the insurance company, including medications and materials.

Variables

Estimation of the total daily costs of a terminal hospitalization and the determination of their difference between the control group and cases were the primary outcomes.

The secondary outcomes were the length of the terminal hospitalization, days in the intensive care unit, the use of IV antibiotics, chemotherapy and radiotherapy in the last month, and the number of costly diagnostic procedures (CT/MRI scans).

Another secondary outcome was the difference between the groups in documenting the fact that the patient was dying. Content analysis of the words referencing the dying phase was approved before data collection by consensus of all three researchers and the project leader.

Time spent in the care of the palliative team was a potential confounder; therefore, it was included in the dataset. Demographic differences were decreased by case-control matching using age, diagnosis, comorbidities, and sex.

The results could have been biased by the data collection methodology and analysis of medical records. For this reason, regular monthly meetings of the researchers and the project manager were scheduled to assure clarification of potential uncertainties and to approve a unified model of data collection. All data were inserted into an Excel chart made for this purpose.

Reporting

The STROBE checklist for case-control studies was used to report the study results. The study protocol was registered with the Technology Agency of the Czech Republic programme ÉTA 3 grant called Dying Matters [TL03000709]. 
Data analysis and statistical methods

The geometric mean and logarithmic transformation of hospital costs were used for power analysis and sample size calculation because of the asymmetric distribution of data. A total of 195 patients in each group were needed to prove the cost difference of $10,000.00$ CZK between the groups with a 0.05 level of significance and a power of 0.8. We used PS Power and Sample Size Calculations (version 3.0).

Standard descriptive statistics were adopted for the description of the data. Numerical variables were described using the mean, standard deviation and 95\% confidence interval. Categorical variables were described using absolute and relative frequencies of categories (percentages).

The statistical significance of differences between the clinical and control groups was tested by Fisher's exact test for categorical variables and by the Mann-Whitney $U$ test for numerical data.

The results were considered statistically significant at the level of alpha $<0.05$ in all applied analyses. Analyses were performed using IBM SPSS Statistics 25.0.0 (IBM Corporation, 2017).

\section{Results}

\section{Participants}

A total of 213 patients who received the support of the palliative care team and 213 controls who died without the support of the palliative care team were included in the study (102 women and 111 men). Eighty-one percent had a main oncologic diagnosis reported on their death certificate, and $19 \%$ had a nononcologic diagnosis. All participants died during the study period, from January 2019 to April 2020. They were grouped according to the Charlson comorbidity index into 3 groups $(0-1,2-4,5$ or more) and matched; therefore, the comorbidity figures in the two groups were the same. 
Table 1

Participant characteristics and length of hospitalization

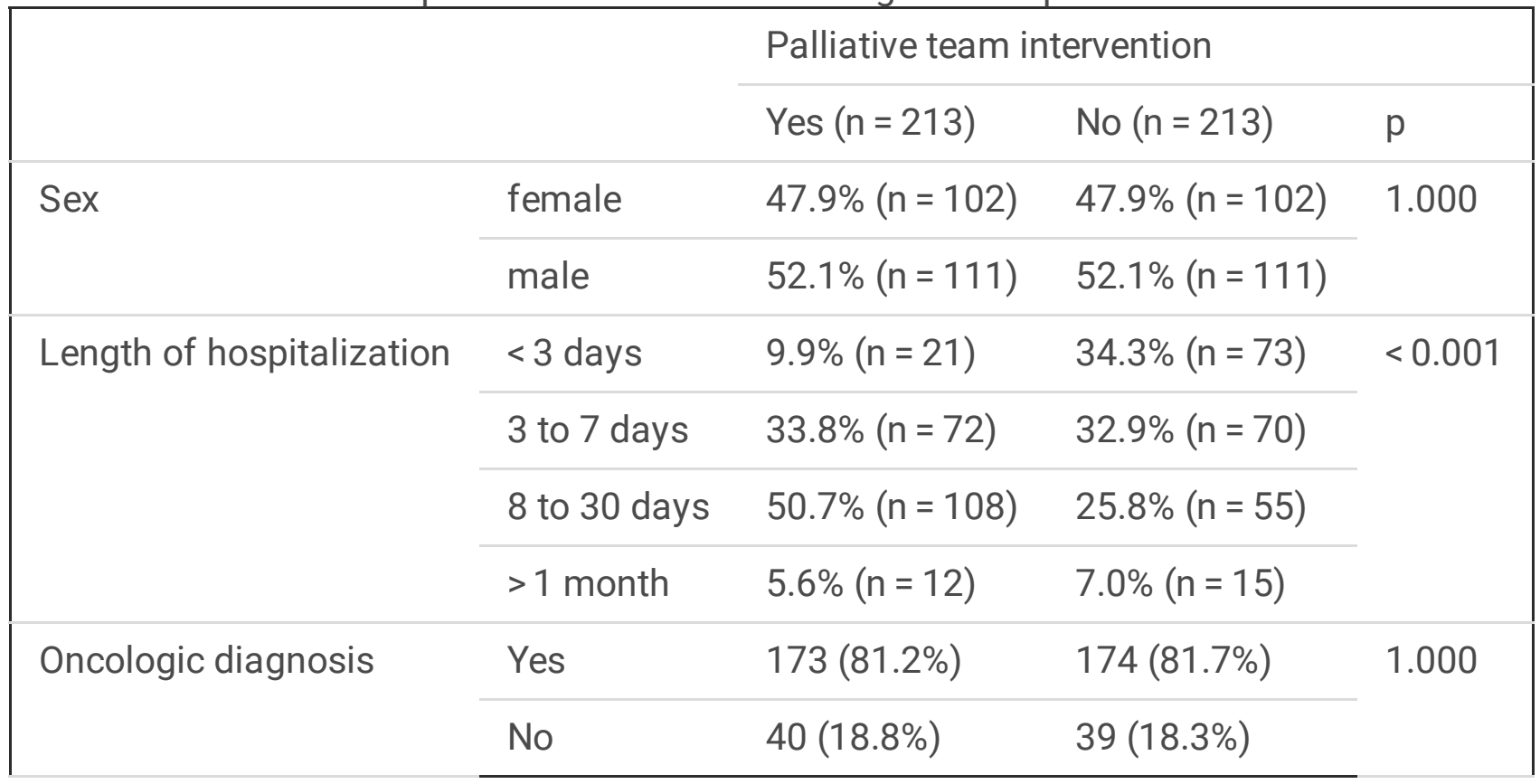

Hospitalization

We proved that there was a significant difference between the groups in the length of terminal hospitalization. Integration of the palliative care team resulted in significantly shorter terminal hospitalizations (mean $=14.3$ days in the palliative care group vs 18.4 in the non-palliative care group, $p \leq$ 0.001). (Table 2)

Patients with palliative care interventions spent significantly less time in the intensive care unit ( $16 \%$ of the hospital days in the palliative care group vs $33 \%$ in the nonpalliative care group, $p \leq 0.001$ ). (Table 2 )

Context of care was clearer in the palliative care group because $62.4 \%$ of the deceased were reported as dying in the medical records, compared to only $30.5 \%$ in the nonpalliative care group ( $\leq 0.001$ ). (Table 3 ) 
Table 2

Differences in costs and costly diagnostic and therapeutic procedures

\section{Palliative team intervention}

Yes $(n=213) \quad$ No $(n=213)$

\begin{tabular}{|c|c|c|c|c|c|c|c|}
\hline & Mean & $\begin{array}{l}\text { Standard } \\
\text { deviation }\end{array}$ & $95 \% \mathrm{Cl}$ & Mean & $\begin{array}{l}\text { Standard } \\
\text { deviation }\end{array}$ & $95 \% \mathrm{Cl}$ & $\mathrm{p}$ \\
\hline $\begin{array}{l}\text { Daily costs } \\
\text { (CZK) }\end{array}$ & 4,392.4 & $4,419.9$ & $\begin{array}{l}3,795.4- \\
4,989.4\end{array}$ & $13,322.8$ & $32,992.8$ & $\begin{array}{l}8,866.6- \\
17,779.0\end{array}$ & $\begin{array}{l}< \\
0.001\end{array}$ \\
\hline $\begin{array}{l}\text { Total costs } \\
\text { (CZK) }\end{array}$ & $64,754.1$ & $124,046.3$ & $\begin{array}{l}47,999.7- \\
81,508.5\end{array}$ & $85,617.2$ & $168,254.4$ & $\begin{array}{l}62,891.8- \\
108,342.6\end{array}$ & 0.558 \\
\hline $\begin{array}{l}\text { Length of } \\
\text { hospitalization }\end{array}$ & 14.27 & 31.43 & $\begin{array}{l}10.03- \\
18.52\end{array}$ & 18.37 & 77.52 & $\begin{array}{l}7.90- \\
28.84\end{array}$ & $\dot{0}_{0.001}$ \\
\hline $\begin{array}{l}\text { Daily doses of } \\
\text { IV antibiotics }\end{array}$ & 1.91 & 1.88 & $\begin{array}{l}1.65- \\
2.16\end{array}$ & 1.78 & 2.17 & $\begin{array}{l}1.49- \\
2.08\end{array}$ & 0.204 \\
\hline $\begin{array}{l}\text { Rate of ICU } \\
\text { days (ICU } \\
\text { days/total days } \\
\text { of terminal } \\
\text { hospitalization) }\end{array}$ & 0.16 & 0.31 & $\begin{array}{l}0.12- \\
0.20\end{array}$ & 0.33 & 0.44 & $\begin{array}{l}0.27- \\
0.39\end{array}$ & $\begin{array}{l}< \\
0.001\end{array}$ \\
\hline $\begin{array}{l}\text { CT or MRI } \\
\text { scans (number } \\
\text { of } \\
\text { scans/maximal } \\
\text { number of } \\
\text { scans in the } \\
\text { group) }\end{array}$ & 0.46 & 0.85 & $\begin{array}{l}0.35- \\
0.58\end{array}$ & 0.60 & 0.96 & $\begin{array}{l}0.47- \\
0.73\end{array}$ & 0.117 \\
\hline
\end{tabular}

Table 3

Differences in oncologic treatment and documentation of the dying phase

\begin{tabular}{|llllll|}
\hline & & \multicolumn{2}{l}{ Palliative team intervention } & P \\
\cline { 2 - 4 } & & Yes $(n=213)$ & No $(n=213)$ & \\
\hline Chemotherapy in the last month & Yes & $3.3 \%(n=7)$ & $6.6 \%(n=14)$ & 0.178 \\
\cline { 2 - 5 } & No & $96.7 \%(n=206)$ & $93.4 \%(n=199)$ & \\
\hline Radiotherapy in the last month & Yes & $2.8 \%(n=6)$ & $4.2 \%(n=9)$ & 0.601 \\
\cline { 2 - 5 } & No & $97.2 \%(n=207)$ & $95.8 \%(n=204)$ & \\
\hline Dying documented in the medical records & Yes & $62.4 \%(n=133)$ & $30.5 \%(n=65)$ & $<0.001$ \\
\hline
\end{tabular}

Cost differences

We calculated all costs of a terminal hospitalization, which was the primary outcome of the study. There was no significant difference between groups in the total costs, but there were more outliers with extremely 
expensive hospitalizations in the nonpalliative group (Fig. 1). The average daily costs were three times lower in the palliative care group $(4,392.4 \mathrm{CZK}=171.3$ EUR per day) than in the nonpalliative care group $(13,992.8 \mathrm{CZK}=545.8$ EUR per day, $p \leq 0.001)$, and there was a significant difference in the daily hospital costs exceeding 10,000.0 CZK ( $p \leq 0.001$ ) (Table 2, Fig. 2).

Chemotherapy and radiotherapy in the last month of life were used similarly, without a significant difference between the cases and the controls (chemotherapy 3.3\% in the palliative care group vs $6.6 \%$ in the non-palliative care group, $p=0.178$, radiotherapy $2.8 \%$ in the palliative care group vs $4.2 \%$ in the nonpalliative care group, $p=0.601$ ) (Table 3 ). We also did not demonstrate any significant difference in IV antibiotic use (1.91 doses/day in the palliative care group vs 1.78 doses/day in the nonpalliative care group, $p=0.201$ ) (Table 2). The usage of diagnostic MRI and CT scans was also similar, and the average number of scans during terminal hospitalization was 0.46 in the palliative care group vs 0.6 in the nonpalliative care group per hospitalization. $(p=0.117)($ Table 2$)$.

\section{Discussion}

Main findings

This study provides clear evidence that integration of the hospital palliative care team during the dying phase can save substantial healthcare costs. We proved that daily costs are three times less expensive in patients supported by a palliative care team. The cost savings are mainly due to the prevention of transfer to the ICU; patients with palliative team support have significantly fewer days spent in the ICU and shorter terminal hospitalizations compared to their matched controls. Moreover, palliative care intervention makes the context of care clear. Twice as many patients in the palliative care group had the dying phase documented in their medical records compared to the nonpalliative care group.

Strengths and limitations

To our knowledge, this is the first study to examine cost savings related to hospital palliative care interventions not only in the Czech Republic but also in the Central European region. The large sample size $(n=213)$ increases the strength of the results. The number of patients was higher than that recommended by power analysis, so a significant difference in primary outcome was reached. Patients of different age groups, cancer types, and noncancer diagnoses were included, and they were properly matched using big national data from registries and propensity scores; therefore, the biases were reduced to a minimum. There is no recommended methodology available for healthcare cost calculations in the dying phase, so we developed our own that accounted for all the costs of procedures billed to the insurance company, medications, materials, and diagnostic procedures. The methodology will be published in detail on the website of the Czech Society of Palliative Medicine and can be used for free by Czech hospitals.

There are also several limitations of the study. The external validity of the study results is limited, as it is a one-centre study and is not international. The retrospective study design limits the richness of data, and especially in the dying phase documentation, it does not mean that when death was not documented in 
the records, it was not discussed with the patients and staff. The semi-qualitative assessment of the dying phase in medical records and the words used to describe the dying process could have been biased by the researchers. We organized monthly meetings of all three researchers and the project leader to clarify discrepancies and to approve a unified method of data collection and content analysis.

Implications for practice

The evidence that hospital palliative care can save a substantial amount of money can help when advocating and negotiating with stakeholders, as hospital palliative care teams are not yet financed by the healthcare system in the Czech Republic and many other countries. It can also be used to support the integration of palliative care in hospitals in middle- and low-income countries. Transfer of patients to the ICU in the dying phase is burdensome and does not provide any benefit. Integration of palliative care in hospital settings can prevent these transfers and allow for a peaceful death.

\section{Future research}

Efforts are ongoing to repeat our study design and to include more hospitals to improve the generalizability and strength of the study. Economic evaluation studies in palliative care are sparse, and more research on the cost-effectiveness of palliative care interventions is still needed.

\section{Conclusion}

Our findings suggest that hospital palliative care teams can help to provide cost-effective end-of-life care. The cost savings are mainly due to the prevention of transfer to the ICU; patients with palliative team support have significantly fewer days spent in the ICU and shorter terminal hospitalizations. Moreover, palliative care intervention clarifies the context of care. The dying phase is more often documented in medical records when patients are supported by the palliative care team.

\section{Abbreviations}

ICU=intensive care unit

IV=intravenous

$\mathrm{MRI} / \mathrm{CR}=$ magnetic resonance imaging/computed tomography

\section{Declarations}

Ethical considerations and consent to participate

This research project was performed in accordance with the Declaration of Helsinki and approved by the Ethics Committee of Faculty Hospital Kralovske Vinohrady in Prague (number EK-VP/62/0/2019). All methods were performed in accordance with approved study protocol and ethical guidelines. Informed consent to participate was obtained from next of kin of deceased patient. 
Consent for publication

Not applicable.

Availability of data and materials

All data generated or analysed during this study are included in this published article and its supplementary information files (Dataset_dying_matters.xlms).

Competing interests

The authors declare that they have no competing interests.

Funding

The authors disclosed receipt of the following financial support for the research, authorship, and publication of this article:

This project was funded by the Technology Agency of the Czech Republic programme ÉTA3 grant called Dying Matters [TL03000709].

Authors' contributions

ZK contributed to the design of the study, led it, secured its funding, and drafted the article. Critical revisions were made by JS, MKB, MM, and HK; JS contributed to case-control matching, statistics, and data analysis; MKB, MM, and $\mathrm{KH}$ collected the data from the records; and PK performed the economic evaluation.

Acknowledgements

The authors would like to thank the Czech Society of Palliative Medicine, particularly Ondrej Slama and Martin Loucka, for their valuable comments and recommendations on the study design and their guarantee of the implementation of the study results in practice. The authors would also like to thank Ivan Rychlik and Martin Havrda, who are the heads of the internal department, for their general support of the research team.

\section{ORCID ID}

Zuzana Kremenova https://orcid.org/0000-0002-5375-6747

Jan Svancara https://orcid.org/0000-0003-1692-3339

Petra Kralova https://orcid.org/0000-0003-2427-1262

Martin Moravec https://orcid.org/0000-0001-6357-3576 
Katerina Hanouskova https://orcid.org/0000-0002-9252-5239

Mayara Knizek-Bonatto https://orcid.org/0000-0002-9659-6560

\section{References}

1. Jahn-Kuch D, Domke A, Bitsche S, et al. End-of-life decision making by Austrian physicians - a crosssectional study. BMC Palliat Care 2020; 19: 4. 2020/01/07

. DOI: 10.1186/s12904-019-0509-3.

2. Dasch B and Zahn PK. Prevalence of therapeutic and diagnostic procedures in the last 14 days of life in hospital patients: a single-center observational study from Germany. Ann Palliat Med 2021 2021/04/10

. DOI: 10.21037/apm-20-2435.

3. May P, Normand C, Cassel JB, et al. Economics of Palliative Care for Hospitalized Adults With Serious IIIness: A Meta-analysis. JAMA Intern Med 2018; 178: 820-829. 2018/05/02

. DOI: 10.1001/jamainternmed.2018.0750.

4. May P, Garrido MM, Cassel JB, et al. Prospective Cohort Study of Hospital Palliative Care Teams for Inpatients With Advanced Cancer: Earlier Consultation Is Associated With Larger Cost-Saving Effect. J Clin Oncol 2015; 33: 2745-2752.

2015/06/10

. DOI: $10.1200 / j c 0.2014 .60 .2334$.

5. May P, Normand C, Del Fabbro E, et al. Economic Analysis of Hospital Palliative Care: Investigating Heterogeneity by Noncancer Diagnoses. MDM Policy Pract 2019; 4: 2381468319866451. 2019/09/20. DOI: $10.1177 / 2381468319866451$.

6. Yadav S, Heller IW, Schaefer N, et al. The health care cost of palliative care for cancer patients: a systematic review. Support Care Cancer 2020; 28: 4561-4573. 2020/05/23. DOI: 10.1007/s00520020-05512-y.

7. Houska A and Loučka M. Patients' Autonomy at the End of Life: A Critical Review. J Pain Symptom Manage 2019; 57: 835-845. 2019/01/07. DOI: 10.1016/j.jpainsymman.2018.12.339.

8. Caissie A, Kevork N, Hannon B, et al. Timing of code status documentation and end-of-life outcomes in patients admitted to an oncology ward. Support Care Cancer 2014; 22: 375-381. 2013/10/01

. DOI: $10.1007 / \mathrm{s} 00520-013-1983-4$.

9. Lewis E, Cardona-Morrell M, Ong KY, et al. Evidence still insufficient that advance care documentation leads to engagement of healthcare professionals in end-of-life discussions: A systematic review.

Palliat Med 2016; 30: 807-824.

2016/03/10

. DOI: $10.1177 / 0269216316637239$.

Page 11/13 
Figures

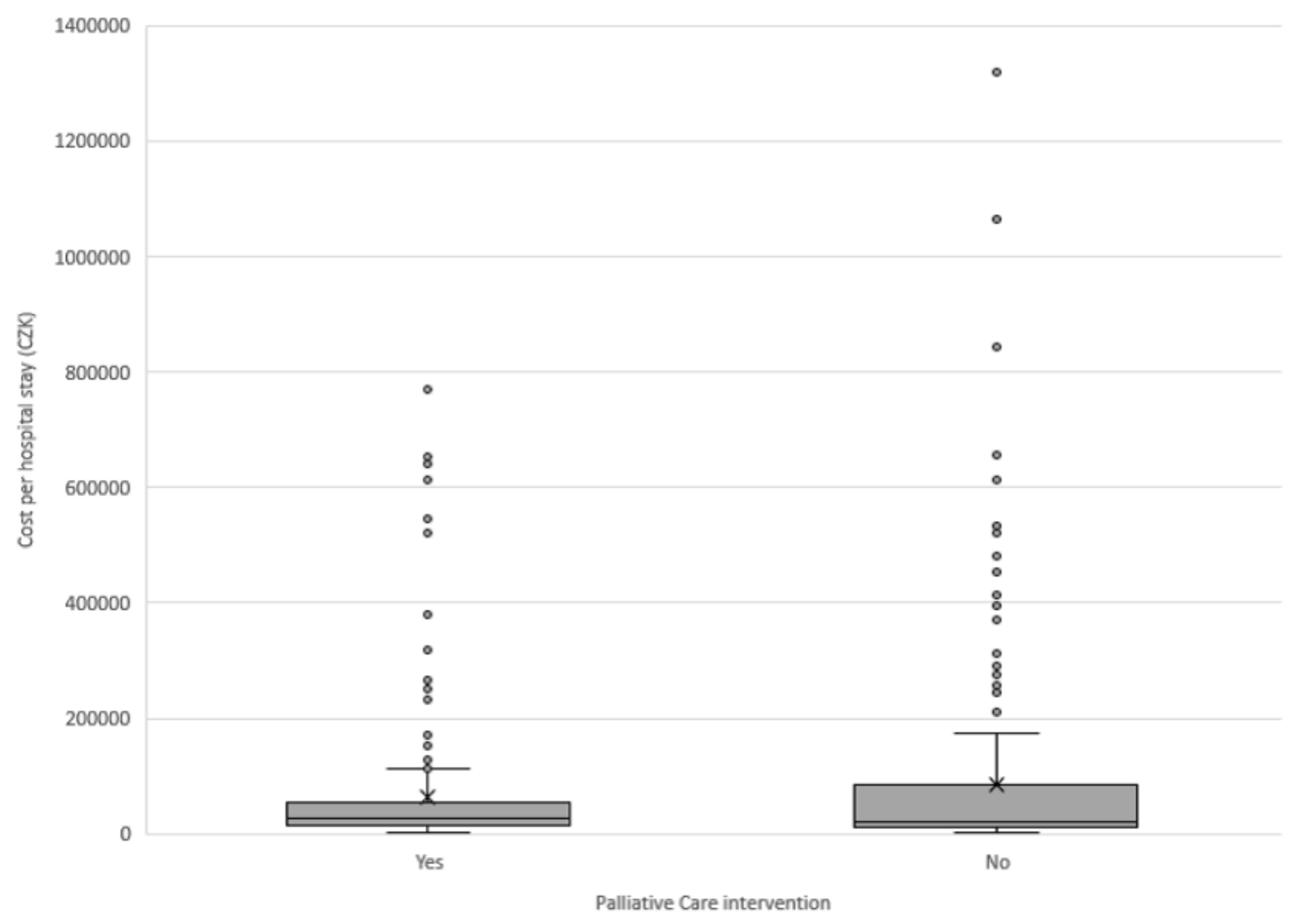

Figure 1

Total costs per terminal hospitalization (in CZK) 
PC intervention - Yes

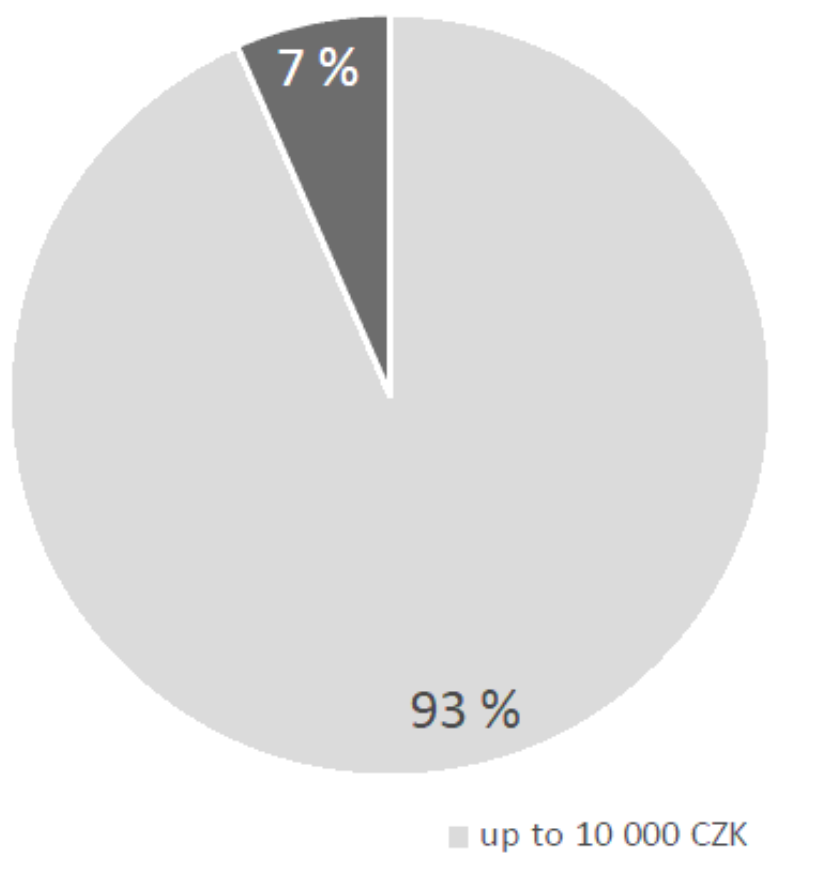

PC intervention - No

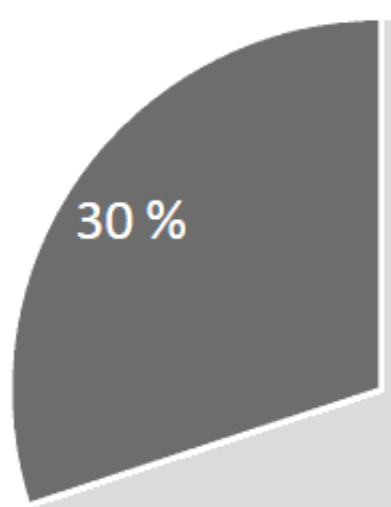

$70 \%$

- over 10000 CZK

Statistical significance between the intervention and control groups was tested by the Fisher exact test $(\mathrm{p}<0.001)$

\section{Figure 2}

Daily hospital costs (in CZK) 